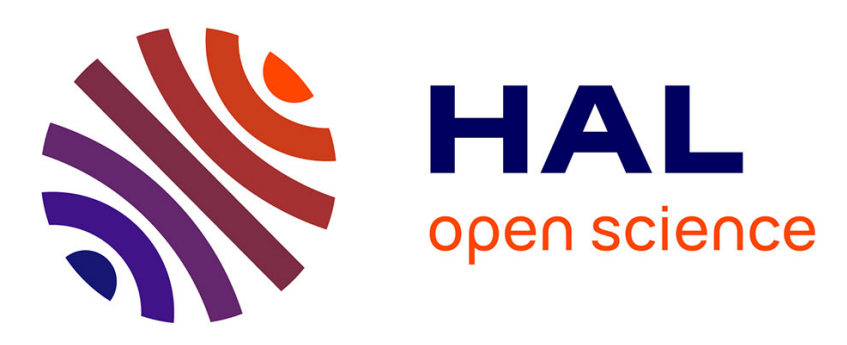

\title{
In situ synthesis of highly crystalline Tb-doped YAG nanophosphor using the mesopores of silica monolith as template
}

Aboulaich Abdelhay, Nathalie Caperaa, Hicham El Hamzaoui, Bruno Capoen, Audrey Potdevin, Mohamed Bouazaoui, Geneviève Chadeyron, Rachid Mahiou

\section{To cite this version:}

Aboulaich Abdelhay, Nathalie Caperaa, Hicham El Hamzaoui, Bruno Capoen, Audrey Potdevin, et al.. In situ synthesis of highly crystalline Tb-doped YAG nanophosphor using the mesopores of silica monolith as template. Journal of Materials Chemistry C, 2015, 3 (19), pp.5041-5049. 10.1039/C5TC00587F . hal-01146369

\section{HAL Id: hal-01146369 https://hal.science/hal-01146369}

Submitted on 1 Dec 2015

HAL is a multi-disciplinary open access archive for the deposit and dissemination of scientific research documents, whether they are published or not. The documents may come from teaching and research institutions in France or abroad, or from public or private research centers.
L'archive ouverte pluridisciplinaire HAL, est destinée au dépôt et à la diffusion de documents scientifiques de niveau recherche, publiés ou non, émanant des établissements d'enseignement et de recherche français ou étrangers, des laboratoires publics ou privés. 
CrossMark \& click for updates

Cite this: J. Mater. Chem. C, 2015, 3, 5041

\section{In situ synthesis of a highly crystalline Tb-doped YAG nanophosphor using the mesopores of silica monoliths as a template $\dagger$}

\author{
Abdelhay Aboulaich, ${ }^{\text {ab }}$ Nathalie Caperaa, ${ }^{\text {ab }}$ Hicham El Hamzaoui, ${ }^{c}$ Bruno Capoen, ${ }^{c}$ \\ Audrey Potdevin, ${ }^{\text {bd }}$ Mohamed Bouazaoui, ${ }^{\text {C }}$ Geneviève Chadeyron*bd and \\ Rachid Mahiou ${ }^{\text {ab }}$
}

\begin{abstract}
This report describes the first synthesis of a highly crystalline YAG:Tb nanophosphor inside the pores of a mesoporous silica monolith (MSM). A simple wet impregnation procedure using a YAG:Tb sol and a MSM platform prepared by a sol-gel method was adopted to prepare highly homogeneous MSMYAG:Tb luminescent composites. The morphological, structural and luminescence properties of in situ generated YAG:Tb nanocrystals, investigated using XRD, TEM, HRTEM, EDS, FTIR, nitrogen gas sorption and photoluminescence measurements, were compared to those of the bulk YAG:Tb phosphor. The specific surface area and the pore volume of the MSM host material were found to be lower when YAG:Tb nanoparticles were grown in the pores of the MSM material. The particle diameter of the nanosized YAG:Tb phosphor was estimated to be about $23 \mathrm{~nm}$. The MSM-YAG:Tb composite exhibited a strong green fluorescence emission with the characteristic main emission band of $\mathrm{Tb}^{3+}$ located at $548 \mathrm{~nm}$. The prepared composite also showed a shorter photoluminescence (PL) lifetime and excitation bands shifted to lower wavelengths compared to the neat YAG:Tb phosphor. The as-prepared MSMYAG:Tb material could be integrated into optoelectronic devices and holds good potential to be applied in decorative lighting.
\end{abstract}

Received 2nd March 2015

Accepted 8th April 2015

DOI: $10.1039 / c 5 t c 00587 f$

www.rsc.org/MaterialsC

\section{Introduction}

Rare earth-doped phosphors (RE-phosphors) attract more and more attention with the continuous emergence of novel and attractive applications in addition to their potential applications in many optoelectronic devices such as light-emitting diodes (LEDs), solid state lasers, optical fibre amplifiers and solar devices. ${ }^{1}$ Among these applications, phosphor-converted LEDs (pc-LEDs) have been extensively investigated during the last few decades due to their potential applications in solid-state lighting and display systems. The great interest in pc-LEDs arises from their

\footnotetext{
${ }^{a}$ Université Clermont Auvergne, Université Blaise Pascal, Institut de Chimie de Clermont-Ferrand, BP 10448, F-63000 Clermont-Ferrand, France

${ }^{b}$ CNRS, UMR 6296, ICCF, F-63171 Aubiere, France

${ }^{c}$ Laboratoire de Physique des Lasers, Atomes et Molécules (PhLAM), CNRS (UMR 8523), CERLA/IRCICA, Université Lille 1-Sciences et Technologies, UFR de Physique, Bâtiment P5, F-59655 Villeneuve D'ASCQ cedex, France

${ }^{d}$ Université Clermont Auvergne, ENSCCF, Institut de Chimie de Clermont-Ferrand, BP 10448, F-63000 Clermont-Ferrand, France.

E-mail: Genevieve.chadeyron@ensccf.fr

$\dagger$ Electronic supplementary information (ESI) available: Fig. S1 and S2 showing high magnification and high resolution TEM images of the ground MSM-YAG:Tb composite. Fig. S3 exhibiting a TEM image of the YAG:Tb crystallized powder. See DOI: $10.1039 / \mathrm{c} 5 \mathrm{tc} 00587 \mathrm{f}$
}

unique properties including but not limited to low power consumption, smaller volume, long lifetime and smaller environmental impact compared with conventional lighting systems. ${ }^{2-4}$ In recent years, pc-LEDs have been broadly applied to screens, traffic lights, automobile lights, LCD backlight, white lighting sources, lighting sources for special purposes, decorative luminaires, etc.

RE-phosphors with a large particle size $(1-20 \mu \mathrm{m})$ are typically combined with LEDs and used as down-converting materials to efficiently convert blue or UV light within the LED into visible light with a longer wavelength. The best-known pc-LED is based on the association of the widely used Ce-doped Yttrium Aluminium Garnet $\mathrm{Y}_{3} \mathrm{Al}_{5} \mathrm{O}_{12}$ (YAG:Ce) phosphor with a blue LED to create white light by combining yellow light from the phosphor with the blue light from a GaN-based LED. RE-phosphors are generally mixed with encapsulant materials, typically with polymeric matrices, and then applied to the LED chip as coatings or films. ${ }^{5}$ Although LED-based lighting offers many advantages over conventional lighting, on average LED lamps remain more expensive than other lighting technologies. One possible way to reduce the packaged pc-LED cost consists of reducing the phosphor cost and/or the required amount of phosphor, which represents about $12 \%$ of the total manufacturing cost of the packaged pc-LED according 
to the report published by the US Department of Energy in September 2013. ${ }^{6}$ In this context, it has been proved in several studies that pc-LEDs using phosphors with a smaller particle size and narrower grain size distribution required a smaller amount of phosphor to obtain an efficiency similar to LEDs using phosphors with bigger particles. ${ }^{7,8}$ This could potentially reduce the cost of pc-LEDs. Other advantages of phosphors with small particle sizes, and more especially nanophosphors, is that the rare earth doping species in the host material are homogeneously distributed compared to the bulk phosphor during their synthesis. This is because nanophosphors are generally prepared using low temperature syntheses starting from molecular precursors in solution yielding high quality phosphor materials since mixing and uniform doping are easy to achieve on a molecular scale., ${ }^{9,10}$ Unlike nanophosphors, bulk phosphor materials are generally prepared, at higher temperatures using a solid state reaction, starting from larger size precursors (typically oxide-based precursors), which generally results in a lower degree of homogeneity, particularly with regard to rare earth doping. Thus, the energy transfer between the luminescent centres within RE-nanophosphors is reduced due to well separated rare-earth dopants in each nanophosphor and, as a result, concentration quenching would be suppressed for nanosized phosphors. ${ }^{11}$

Consequently, research on nanophosphors has attracted broad interest for use in pc-LEDs in the past few decades. ${ }^{9,12-18}$ Otherwise, the development of new processes allowing the production of encapsulating materials and supports for nanophosphor materials with high and easy control of the final macroscopic shape (including size and morphology) is of great interest now especially for decorative lighting. For this purpose, the mesoporous silica monoliths (MSMs) might be a potential candidates because of their unique properties such as rigidity, high surface area, tuneable pore diameter, and photo/thermal and chemical stability. ${ }^{19}$ Indeed, the high surface area and the tuneable pore diameter provided by mesoporous silica matrices should allow better dispersion of the RE-nanophosphor with a narrow size distribution and achieve highly homogeneous nanophosphor/silica optical materials. In addition, the control of shape and size of the final MSM can be easily achieved by using a suitably shaped-recipient during the gelation/consolidation stage of the process. These features should enable new LED lighting devices designed for decorative purposes while reducing the required amount of the phosphor.

Ordered mesoporous silica sieves like MCM41 and SBA15 have been widely used as host matrices for various luminescent materials including rare earth complexes, rare earth metal oxides, rare earth fluorides, dye molecules and quantum dots. ${ }^{20-32}$

However, ordered mesoporous silica sieves require relatively expensive surfactants and triblock copolymers (e.g. P123, F127) used as structure-directing agents. Furthermore, it is often difficult to prepare bulk materials from those ordered silica sieves. Silica xerogels with a tuneable pore diameter have also been synthesized, using alkoxide-based sol-gel methods, to yield stable bulk silica monoliths without using any structuredirecting agents. ${ }^{31}$ These materials may have more commercial potential because of low-cost and widely available silica precursors and reactants.
Mesoporous silica embedded with luminescent compounds can be prepared by using several approaches. One approach consists of introducing the luminescent material into the silica matrix during the early stages of preparation by using a co-condensation method. This one-step synthesis method yields stable hybrid luminescent materials with strong interactions (usually with covalent bonds) between the host silica framework and the luminescent compounds, thus reducing the leaching of guest compounds from the silica matrix. ${ }^{31}$ However, it is recognized that the difference in reaction rates between silica and guest precursors might lead to non-homogeneous dispersion of luminescent compounds and thus phase separation can occur. ${ }^{21}$ In addition, a decrease in emission intensity is often observed in the final hybrid material compared to the pure luminescent material. ${ }^{29}$ The second approach is based on the incorporation of luminescent compounds once the silica framework is formed. This method has been widely used for the in situ synthesis of a large number of metal oxides and semiconductor nanoparticles in the pores of mesoporous silica. ${ }^{33-36}$ However, very few reports are available on the in situ synthesis of RE-nanophosphors, especially RE-doped YAG nanophosphors, inside the pores of the MSM. Among RE-doped YAG, the $\mathrm{Tb}^{3+}$-doped YAG (YAG:Tb) phosphor has been extensively studied due to its well-known green emission, which can be efficiently sensitized by means of several sources such as cathode-ray, VUV, UV and low-voltage excitations. ${ }^{37}$

Herein, we report the first in situ synthesis of YAG:Tb nanocrystals inside the pores of the MSM using a simple wet impregnation procedure followed by a calcination stage to achieve a highly crystalline YAG phase. For comparison purposes, YAG:Tb crystalline powder was also prepared under the same conditions as the MSM-YAG:Tb nanocomposite.

\section{Experimental section}

\section{Synthesis of mesoporous silica monoliths (MSMs)}

Monolithic porous silica matrices were prepared following the procedure already reported. ${ }^{38}$ This sol-gel process yields a large range of mesoporous xerogels with interconnected pores and a well-controlled pore size distribution. In this paper, we consider silica xerogels with a mean pore size of $24 \mathrm{~nm}$. These xerogels were obtained by hydrolysis and polycondensation of tetraethylorthosilicate (TEOS), followed by a drying step and stabilization in air at $1000{ }^{\circ} \mathrm{C}$.

\section{Synthesis of YAG:Tb sol}

YAG:Tb (20 mol\%) sol was prepared according to the method already detailed in our previous reports. ${ }^{37,39}$ Anhydrous yttrium chloride $\mathrm{YCl}_{3}$ (99.99\% pure, Aldrich), terbium chloride $\mathrm{TbCl}_{3}$ (99.99\% pure, Aldrich), metallic potassium (98\% pure, Aldrich), aluminium isopropoxide (99.99+\% pure, Aldrich) and anhydrous isopropanol (iPrOH, 99.8+\% pure, Aldrich) have been used as starting materials. The synthesis consists of preparing separately a solution A of anhydrous yttrium and terbium chlorides dissolved in anhydrous isopropanol and a solution B of potassium isopropoxide. Solution B is slowly added to solution A under 
vigorous stirring, a precipitate of $\mathrm{KCl}$ appears immediately. The mixed solution is maintained at $85{ }^{\circ} \mathrm{C}$ for $1 \mathrm{~h}$. Then, a known quantity of aluminium isopropoxide powder is added directly into the A-B mixture. After further refluxing for $4 \mathrm{~h}$ at $85{ }^{\circ} \mathrm{C}$, a clear and homogeneous solution (solution $\mathrm{C}$ ) is obtained together with the $\mathrm{KCl}$ precipitate. The latter is removed by centrifugation after cooling. The centrifugation step was repeated three times in order to make out all traces of $\mathrm{KCl}$ salts. The obtained clear supernatant corresponds to the YAG:Tb sol.

\section{In situ synthesis of YAG:Tb nanocrystals into the pores of mesoporous silica monoliths (MSM-YAG:Tb)}

The MSM was dried under vacuum for $12 \mathrm{~h}$ at $100{ }^{\circ} \mathrm{C}$ before use. YAG:Tb nanocrystals were synthesized in the pores of the MSM using a simple wet impregnation process. In a typical procedure, a dried MSM was immediately placed in a YAG:Tb sol for $72 \mathrm{~h}$. To complete the pore filling, the imbibed MSM was then dried in an oven at $80{ }^{\circ} \mathrm{C}$ under vacuum for $12 \mathrm{~h}$ and then placed again in YAG:Tb sol for $10 \mathrm{~min}$. The $10 \mathrm{~min}$ impregnation process was repeated 10 times. Each impregnation step is followed by a drying step at $80{ }^{\circ} \mathrm{C}$ for $15 \mathrm{~min}$ to remove the isopropanol solvent. The imbibed MSM was finally sintered at $1100{ }^{\circ} \mathrm{C}$ for $4 \mathrm{~h}$ to let the YAG:Tb crystals grow inside the mesopores of the silica matrix. This material will be named MSM-YAG:Tb in the next part of this report. The in situ preparation process of YAG:Tb nanocrystals in the pores of the MSM is illustrated in Scheme 1.

In order to quantify the amount of YAG:Tb nanocrystals loaded into the silica monolith, the silica template was removed from the MSM-YAG:Tb nanocomposite using an aqueous solution of $\mathrm{NaOH}(1 \mathrm{M})$. A sample of MSM-YAG:Tb was immersed in the basic solution and heated at $55{ }^{\circ} \mathrm{C}$ overnight with vigorous stirring. After silica dissolution, the YAG:Tb nanocrystals were subsequently collected as a powder by centrifugation (10000 rpm/10 $\mathrm{min})$.

\section{Synthesis of YAG:Tb crystalline powder}

To conduct a comparative study, we separately prepared YAG:Tb crystalline powder. The YAG:Tb sol was hydrolyzed by introducing an excess of water, resulting in a transparent gel, which was further dried at $80{ }^{\circ} \mathrm{C}$ to yield a white xerogel. The latter was sintered at $1100{ }^{\circ} \mathrm{C}$ for $4 \mathrm{~h}$ in a muffle furnace in order to obtain well-crystallized YAG:Tb phosphor powder.

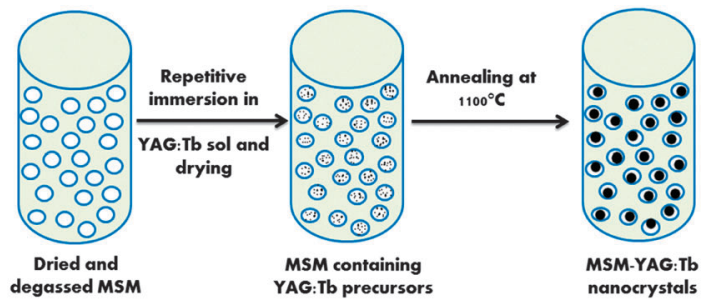

Scheme 1 Schematic illustration of the in situ formation of YAG:Tb nanocrystals in the pores of MSM.

\section{Characterization}

The XRD measurements were performed using a Philips Xpert Pro diffractometer using $\mathrm{Cu} \mathrm{K} \alpha$ radiation with a wavelength of $1.5406 \AA$. Transmission Electron Microscope (TEM) images were taken by grinding a small piece of MSM-YAG:Tb into powder and by depositing the powder onto a carbon filmsupported copper grid. The samples were studied using a FEI Tecnai G2 20 instrument operating at $200 \mathrm{kV}$. In order to characterize the porosity of the MSM and MSM-YAG:Tb, nitrogen sorption isotherms were recorded at 77.35 K using a Quantachrome porosimeter Autosorb 1-LP-MP, after an outgassing process of several hours at $150{ }^{\circ} \mathrm{C}$ under secondary vacuum. The specific surface area was determined by the Brunauer-Emmett-Teller (BET) method. ${ }^{40}$ The pore size distribution was obtained from the desorption isotherm using the Barrett-Joyner-Halenda (BJH) model. ${ }^{41}$ Photoluminescence (PL) emission and excitation features, as well as absolute photoluminescence quantum yield (PL-QY) values, were measured using a C9920-02G PL-QY measurement system from Hamamatsu. The setup comprises a $150 \mathrm{~W}$ monochromatized Xe lamp, an integrating sphere (Spectralon coating, $\varnothing=3.3$ inch) and a high sensitivity CCD spectrometer for detecting the whole spectral luminescence. Photoluminescence excitation (PLE) spectra were obtained by exciting the samples from 250 to $400 \mathrm{~nm}$ with a $3 \mathrm{~nm}$ increment and measuring their absolute PL QY by monitoring the overall emission of the samples in the $400-700 \mathrm{~nm}$ wavelength range. Absolute QY values were then used and combined with the absorption coefficient (also measured using the apparatus) to plot the excitation spectra. The PL decays were obtained using a pulsed dye laser (Continuum ND60) pumped by a frequency doubled pulsed YAG: $\mathrm{Nd}^{3+}$ laser (continuum surelite I) with $10 \mathrm{~ns}$ pulse, $0.1 \mathrm{~cm}^{-1}$ band-width and $10 \mathrm{~Hz}$ repetition rate. The dye laser was followed by a KDP frequency doubler. The UV beam is isolated from the fundamental dye laser beam using a Pellin-Broca prism associated with an iris diaphragm and a UV Bandpass filter UG-11. Rhodamine 590 used as a dye solution provided energy up to $400 \mu \mathrm{J}$ in the doubled selected UV region. Fluorescence decays were then measured using a LeCroy $400 \mathrm{MHz}$ digital oscilloscope.

\section{Results and discussion}

The $\mathrm{N}_{2}$ adsorption-desorption isotherms and pore-size distributions of the as-prepared MSM before loading with YAG:Tb precursors are shown in Fig. 1.

From Fig. 1(a), it can be seen that the MSM host has a type IV isotherm with a $\mathrm{H}_{2}$ hysteresis loop according to the IUPAC classification, which is characteristic of mesoporous materials with interconnected pores. The pore size distribution is given by the derivative of the desorbed volume as a function of the pore diameter (Fig. 1(b)). The MSM material exhibited interconnected pores with an average diameter of $24 \mathrm{~nm}$. The specific surface area was found to be around $130 \mathrm{~m}^{2} \mathrm{~g}^{-1}$. The total pore volume, measured at the highest relative pressure $\left(P / P_{0}\right)$, is $0.57 \mathrm{~cm}^{3} \mathrm{~g}^{-1}$ (Table 1$)$. Compared to the non-embedded 

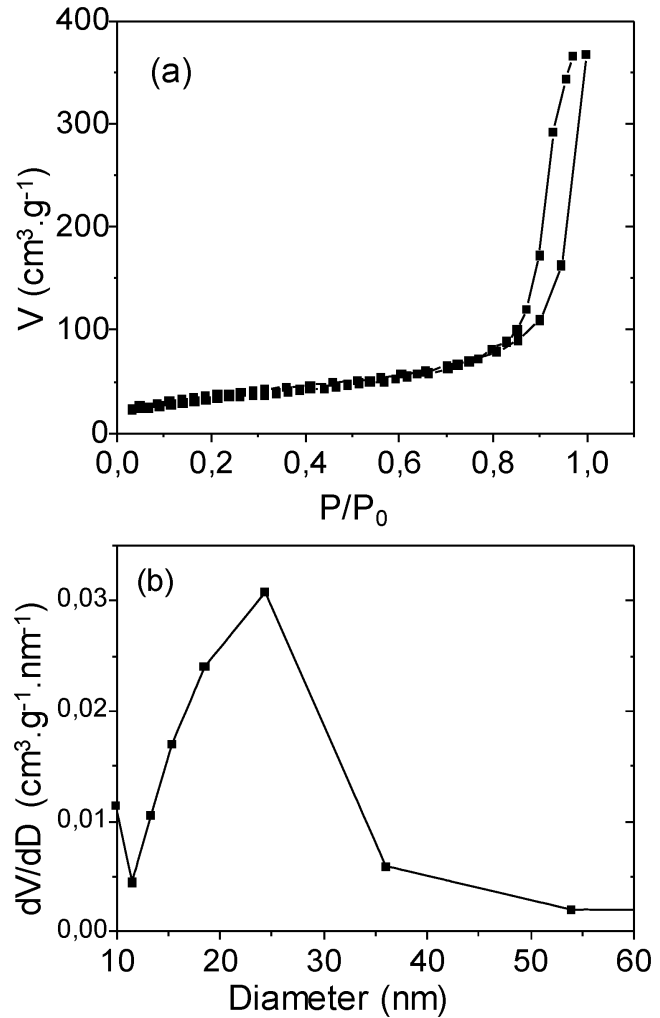

Fig. 1 (a) $\mathrm{N}_{2}$-sorption isotherm and (b) BJH pore size distribution of the as-prepared MSM.

Table 1 Specific surface area and pore volume of MSM and MSM-YAG:Tb materials

\begin{tabular}{lrl}
\hline Sample & Specific surface area $\left(\mathrm{m}^{2} \mathrm{~g}^{-1}\right)$ & Pore volume $\left(\mathrm{cm}^{3} \mathrm{~g}^{-1}\right)$ \\
\hline MSM & 130 & 0.57 \\
MSM-YAG:Tb & 60 & 0.1
\end{tabular}

MSM, the MSM-YAG:Tb composite showed much lower specific surface area and pore volume. The drop in the specific surface area (by about $54 \%$ ) and pore volume (by about $80 \%$ ) within the MSM-YAG:Tb material is in agreement with an efficient pore filling with the YAG:Tb phosphor by using our wet impregnation procedure. The silica template was successfully removed from the composite using concentrated $\mathrm{NaOH}$ solution as described in the Experimental section. It was found that the YAG:Tb content in the composite represents about $10-15 \mathrm{wt} \%$ relative to silica.

The MSM annealed at $1100{ }^{\circ} \mathrm{C}$ for $4 \mathrm{~h}$, MSM-YAG:Tb and YAG:Tb powders have been characterized using XRD. The resulting patterns (Fig. 2) show that MSM-YAG:Tb and YAG:Tb powders are crystallized while the neat MSM annealed at the same temperature is amorphous. All diffraction peaks of both MSM-YAG:Tb and YAG:Tb powders are assigned to the YAG structure (JCPDS-file 33-0040). The XRD pattern of MSMYAG:Tb also shows a broad component at about $20^{\circ}$ attributed to the amorphous silica matrix.

In the first approximation, the average crystallite size has been determined from the full-width at half maximum (FWHM)

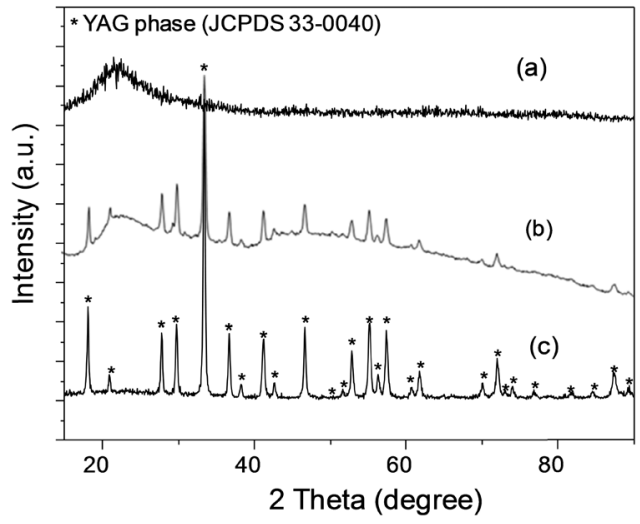

Fig. 2 XRD patterns of the (a) MSM annealed at $1100{ }^{\circ} \mathrm{C} / 4 \mathrm{~h}$, (b) MSMYAG:Tb and (c) YAG:Tb powder.

of the diffraction peaks using Scherrer's equation: $\langle D\rangle=k \lambda$ / $\beta \cos \theta$, where $D$ is the mean particle size, $k$ is a geometric factor (here, $k=0.9),{ }^{42} \lambda$ is the X-ray wavelength $(1.5406 \AA$ ), and $\beta$ is the corrected half-width of the selected diffraction peak located at about $33^{\circ}(2 \theta)$. The average crystallite sizes of the in situ prepared YAG:Tb particles are about $23 \mathrm{~nm}$ as estimated from the formula. This crystallite size matches well with the pore diameter of the MSM, thus indicating that YAG:Tb nanocrystals are mainly formed inside the pores of the mesoporous material. To the best of our knowledge, this is the first demonstration of YAG:Tb nanophosphor synthesis using the pores of the MSM as nanoreactors.

The morphological and structural properties as well as the chemical composition of the MSM-YAG:Tb composite were also investigated using TEM and HR-TEM analyses coupled with EDS measurements (Fig. 3). The TEM image in dark field mode (Fig. 3(b)) reveals the coexistence of bright (i.e. diffracting zones) and dark areas (i.e. non-diffracting zones) corresponding to the crystallized and amorphous areas, respectively. Moreover, EDS analysis performed on a whole powder grain (Fig. 3(c)) shows the coexistence of a small amount of YAG:Tb in a matrix logically dominated by silica while the EDS spectrum from strongly crystallized areas shows YAG:Tb enriched composition of these zones of a few tens of nanometers. In addition, bright areas seem to be relatively well distributed within the amorphous silica enriched zones, indicating a relatively high homogeneous dispersion of crystallized YAG:Tb in the MSM host material. The HR-TEM image (inset of Fig. 3(b)) shows the distances between the adjacent lattice fringes to be $3.24 \AA$, a value consistent with the spacing of (321) planes in the YAG cubic structure. $^{43}$

Besides, HR-TEM images taken on the ground MSM-YAG:Tb composite (Fig. S1, ESI $\dagger$ ) clearly show discernible YAG:Tb nanoparticles with a spherical shape and the particle diameter in the range of 20-30 nm, in agreement with the crystallite size calculated from the XRD pattern. These results confirm that highly crystalline YAG:Tb nanophosphors are synthesized in situ inside the pores of the MSM and that YAG:Tb nanoparticles are monocrystalline. In contrast, the TEM image of the YAG:Tb crystalline powder (Fig. S3, ESI $\dagger$ ) actually shows highly 

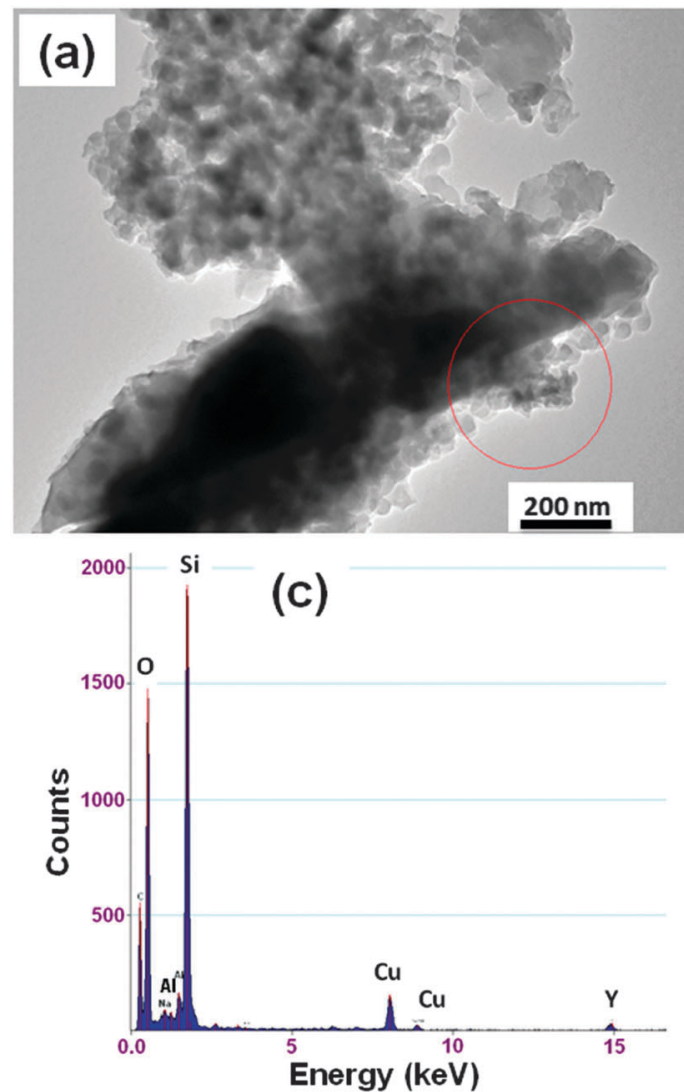

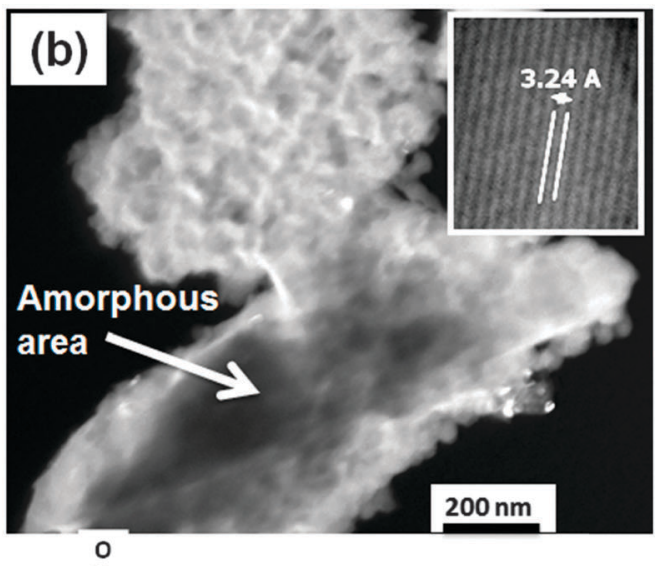

(d)

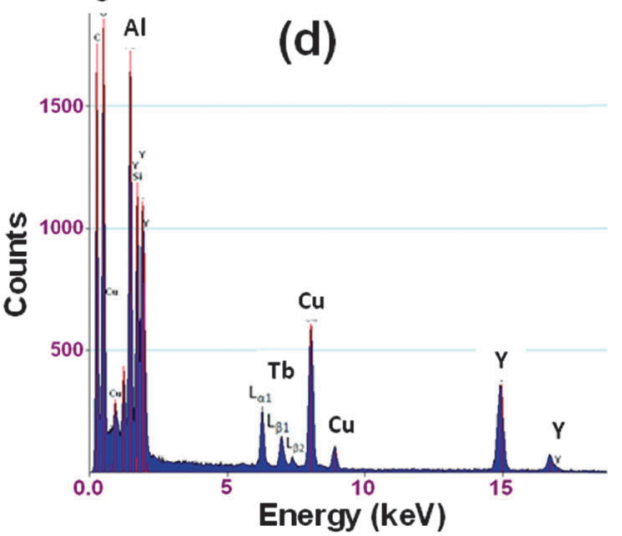

Fig. 3 TEM image acquired in (a) bright field mode and (b) dark field mode. The EDS spectrum corresponding to (c) an extended zone and (d) a local crystallized zone of the MSM-YAG:Tb composite. Inset of (b) shows the HR-TEM image of a crystallized area.

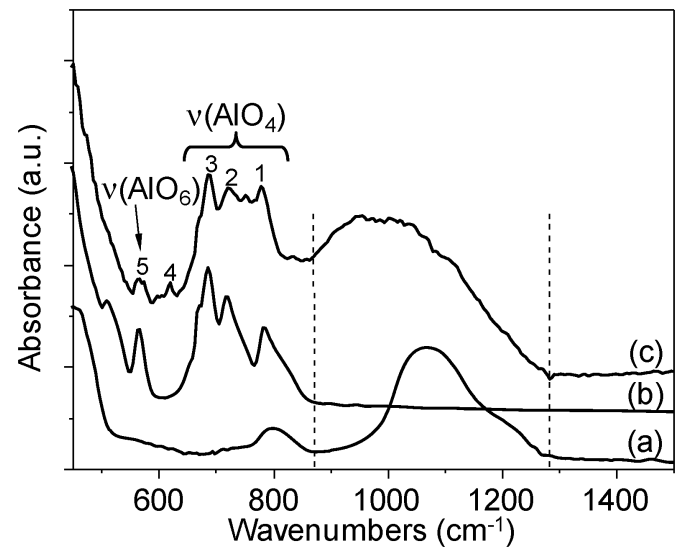

Fig. 4 FT-IR spectra of the (a) MSM, (b) YAG:Tb phosphor powder and (c) MSM-YAG:Tb composite.

agglomerated particles with a mean particle size in the range 60-80 nm.

The FT-IR spectrum of the MSM-YAG:Tb (Fig. 4(c)) composite shows absorption bands at 785, 728 and $693 \mathrm{~cm}^{-1}$ (bands 1 , 2 and 3), characteristic of the stretching vibrations of $\mathrm{AlO}_{4}$ tetrahedra in the $\mathrm{Y}_{3} \mathrm{Al}_{5} \mathrm{O}_{12}$ cubic structure. ${ }^{44,45}$ The absorption band at about $570 \mathrm{~cm}^{-1}$ (band 5) is attributed to the stretching vibration of $\mathrm{AlO}_{6}$ octahedra. ${ }^{45}$ In addition to these absorption bands, the MSM-YAG:Tb composite shows an absorption peak at about $623 \mathrm{~cm}^{-1}$ (band 4) which was not observed in the YAG:Tb powder. This peak is also due to the Al-O stretching vibration. It could be noticed that this peak was already observed in RE-doped YAG phosphors with the particle size smaller than $40 \mathrm{~nm}$ and could result from the surface states-related $\mathrm{Al}$ species when the surface/volume ratio of the YAG particles is particularly high. ${ }^{9}$ Based on these results, it can be concluded that YAG:Tb nanocrystals are formed within the MSM matrix.

By the way, the FT-IR spectrum of the MSM-YAG:Tb nanocomposite also revealed a broader absorption band with a shift to low wavenumbers in the $870-1200 \mathrm{~cm}^{-1}$ region when compared to that of the MSM. This change most likely results from mixed bands due to $\mathrm{Si}-\mathrm{O}-\mathrm{Si}$ and $\mathrm{Si}-\mathrm{O}-\mathrm{Al}$ linkages. ${ }^{46,47}$ The Si-O-Al bond could actually result from the condensation reaction between $\mathrm{Si}-\mathrm{OH}$ monolith surface groups and $\mathrm{Al}-\mathrm{OH}$ groups on the surface of YAG:Tb nanocrystals.

Fig. 5 shows emission and excitation spectra of MSMYAG:Tb nanocrystals. The emission and excitation spectra of YAG:Tb phosphor powder are also shown for comparison. Both the YAG:Tb powder and the MSM-YAG:Tb nanocomposite exhibit four main PL emission bands, associated with the $\mathrm{f}-\mathrm{f}$ internal transition from the ${ }^{5} \mathrm{D}_{4}$ state to the ${ }^{7} \mathrm{~F}_{J}(J=6,5,4,3,2)$ states of $\mathrm{Tb}^{3+}$ ions in a crystallized YAG matrix. ${ }^{48}$

Due to the cross-relaxation between ${ }^{5} \mathrm{D}_{3}{ }^{5} \mathrm{D}_{4}$ and ${ }^{7} \mathrm{~F}_{0}{ }^{7} \mathrm{~F}_{6}$ of two $\mathrm{Tb}^{3+}$, the blue emission of ${ }^{5} \mathrm{D}_{3} \rightarrow{ }^{7} \mathrm{~F}_{J}$ is not observed at this 


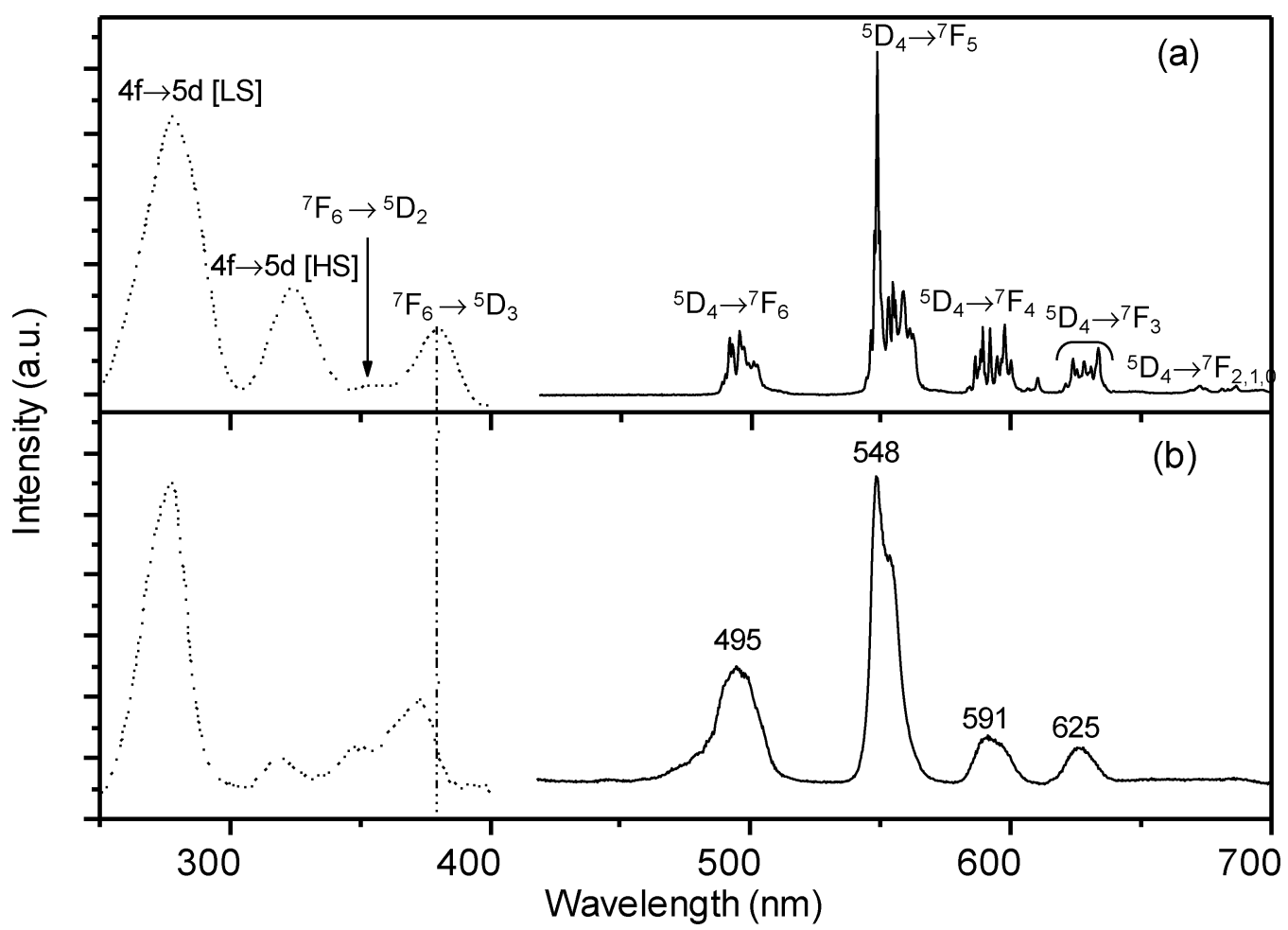

Fig. 5 Room temperature PL emission (solid line) and PLE (dotted line) spectra of the (a) YAG:Tb phosphor powder and (b) MSM-YAG:Tb nanocomposite. Emission spectra were obtained upon $375 \mathrm{~nm}$ excitation. Excitation spectra were recorded with an emission wavelength at $547 \mathrm{~nm}$. From Table 2 and Fig. 5, it can be observed that the excitation bands of the MSM-YAG:Tb composite are shifted to shorter wavelengths compared to YAG:Tb crystallized powder. Such an effect has already been reported for the nanocrystalline YAG:Tb ${ }^{3+}$ phosphor where a blue-shift is observed for the lowest $4 f-5 d$ band when compared with the bulk YAG:Tb ${ }^{3+}$ phosphor. ${ }^{57}$ This is because the excited states such as $5 d$ are affected by the surrounding electronic shell. In other words, the $5 \mathrm{~d}$ electrons have a strong interaction with the neighbouring anion ligands in the compound.

doping concentration. ${ }^{49}$ For both samples, the ${ }^{5} \mathrm{D}_{4} \rightarrow{ }^{7} \mathrm{~F}_{5}$ transition $(548 \mathrm{~nm})$ is most intense. Moreover, it can be seen from Fig. 5 that the PL emission bands of the YAG:Tb crystallized powder are characterized by well-resolved Stark lines, in accordance with the previously reported studies. ${ }^{44}$ In the case of the MSM-YAG:Tb nanocomposite, the emission spectra are almost identical to that of the YAG:Tb crystallized powder but the bands are broader and more characteristic of $\mathrm{Tb}^{3+}$ ions dispersed in nano-sized YAG particles. ${ }^{50,51}$

This broadening is generally ascribable to the nanometersize of the YAG particles. It can be firstly attributed to the fact that fluorescence mainly arises from $\mathrm{Tb}^{3+}$ ions located on the nanocrystal surface. The crystallographic sites of these ions are disrupted, leading to a very slightly modified PL spectrum when compared with that of regular sites. The shape of the emission bands is also related to the fact that $\mathrm{Tb}^{3+}$ ions are embedded in a glass ceramic, resulting in a further inhomogeneous broadening, due to the random stress experienced by the YAG nanocrystals in the pores of the silica matrix. It is to be mentioned that similar emission spectra were reported when YAG:Tb nanocrystals were embedded in the silica xerogel. ${ }^{52}$

It could be noticed that no wavelength shift was observed when comparing emission bands of MSM-YAG:Tb with those of the YAG:Tb phosphor powder. The main emission band located at $548 \mathrm{~nm}$ gave rise to the well-known green luminescence of
Table 2 Excitation wavelength positions (in $\mathrm{nm}$ ) and the corresponding transitions of the YAG:Tb powder and the MSM-YAG:Tb composite

\begin{tabular}{llllr}
\hline Level & $5 \mathrm{~d}^{1}[\mathrm{LS}]$ & $5 \mathrm{~d}^{1}[\mathrm{HS}]$ & ${ }^{5} \mathrm{D}_{2}$ & ${ }^{5} \mathrm{D}_{3}$ \\
\hline YAG:Tb powder & 278 & 325 & 355 & 380 \\
MSM-YAG:Tb & 277 & 319 & 348 & 372
\end{tabular}

$\mathrm{Tb}^{3+}$ in both cases. The excitation spectra of both samples consist of, at least, three bands in the $250-400 \mathrm{~nm}$ wavelength range. The two bands located at a shorter excitation wavelength of both the YAG:Tb phosphor powder and the MSM-YAG:Tb composite are attributed to the $4 \mathrm{f} \rightarrow 5 \mathrm{~d}\left(4 \mathrm{f}^{8} \rightarrow 4 \mathrm{f}^{7} 5 \mathrm{~d}^{1}\right)$ spinallowed transition (known as low-spin [LS] transition) and spinforbidden transition (known as high-spin [HS] transition) of $\mathrm{Tb}^{3+},{ }^{33,54}$ while the excitation bands located at a longer excitation wavelength can be ascribed to the ${ }^{7} \mathrm{~F}_{6} \rightarrow{ }^{5} \mathrm{D}_{2}$ and ${ }^{7} \mathrm{~F}_{6} \rightarrow{ }^{5} \mathrm{D}_{3}$ transitions,${ }^{55}$ as shown in Table 2.

Moreover, the $5 \mathrm{~d}$ energy level has a close relationship with the conduction band of the compound. Indeed, due to quantum confinement, smaller crystallites have a wider energy band gap. This leads to the $5 \mathrm{~d}$ energy level change, which is reflected in the blue-shift of the energy of the $4 \mathrm{f}-5 \mathrm{~d}$ level due to this size effect. ${ }^{56}$

Fig. 6 displays a photograph of a sculpted $30 \times 100 \mathrm{~mm}$ piece of MSM-YAG:Tb, before and after excitation at $375 \mathrm{~nm}$, which shows bright and highly homogeneous green emission, 


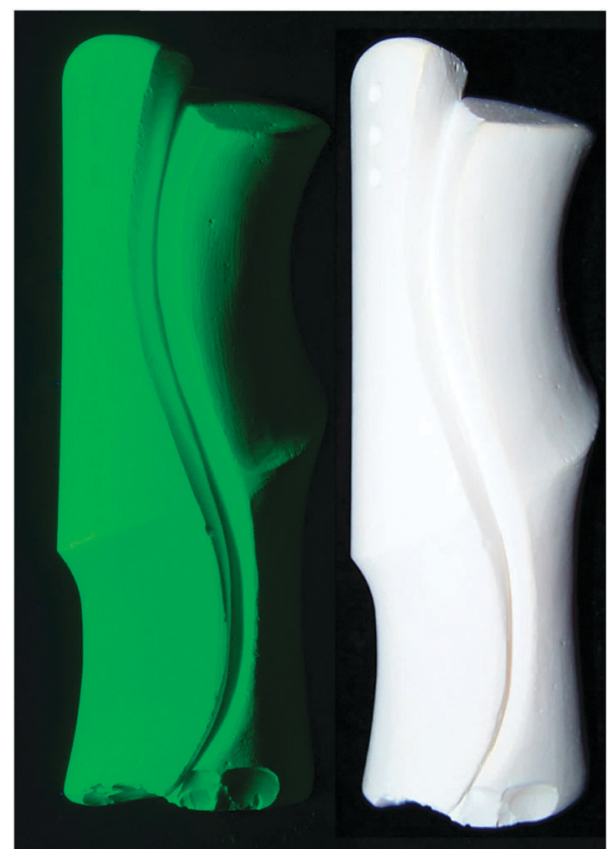

Fig. 6 Photograph of the sculpted piece of the MSM-YAG:Tb composite with a width/height dimension of 30/100 mm before (right) and after (left) $375 \mathrm{~nm}$ excitation.

in accordance with the homogeneous distribution of YAG:Tb nanocrystals in the silica matrix as shown by TEM images. The MSM-YAG:Tb composite can be easily adorned with a LED source to make green luminescent objects with various shapes and sizes for innovative decorative purposes. This approach could also be generalized and applied to various luminescent materials in order to tailor decorative objects depending on the desired colour and shape.

As the YAG:Tb particles in the MSM host are nanosized and are strongly affected by the surrounding medium, its related PL lifetime is expected to be shorter because the probability of nonradiative recombinations increase in nanocrystals compared to bulk materials, as already reported in the literature for other RE-doped phosphors or phosphors embedded in silica media. ${ }^{58-61}$

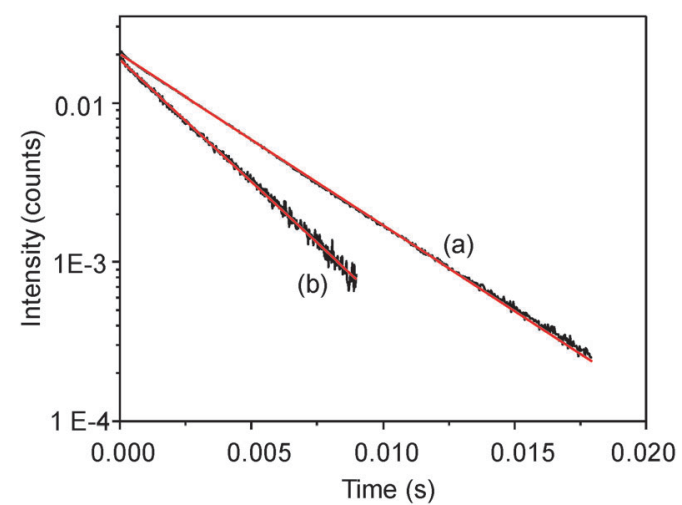

Fig. 7 PL decay curves of $548 \mathrm{~nm}$ emission of the (a) YAG:Tb powder and (b) MSM-YAG:Tb nanocomposite recorded upon $375 \mathrm{~nm}$ excitation at room temperature. Red line represents the mono-exponential fit.
To confirm this statement, PL decay of the most intense emission wavelength of the $\mathrm{Tb}^{3+}$ ions (i.e. $548 \mathrm{~nm}$ ) in the YAG:Tb powder and the MSM-YAG:Tb nanocomposite was both recorded and fitted to a mono-exponential function, as shown in Fig. 7. Using these fitted curves, the $\mathrm{Tb}^{3+}$ emission lifetimes were calculated to be about 4 and $2.8 \mathrm{~ms}$ in the YAG:Tb powder and MSM-YAG:Tb nanocomposite, respectively. This result is thus consistent with our previous statement. It is also in line with PL QY measurements upon excitation at $278 \mathrm{~nm}$, which obviously show lower PL QY in the MSM-YAG:Tb composite compared to YAG:Tb powder (about 10\% and 35\% for MSM-YAG:Tb and YAG:Tb powder, respectively). The shorter PL decay time observed with the MSM-YAG:Tb composite therefore supports our assertion that the nanosized YAG:Tb phosphor is formed in situ in the MSM matrix where YAG:Tb particles have a higher surface/ volume ratio than YAG:Tb particles in the phosphor powder.

\section{Conclusions}

In this report, we have demonstrated a successful in situ synthesis of a highly crystalline YAG:Tb nanophosphor using the pores of the MSM as nano-reactors via a simple wet impregnation procedure. The MSM host material with a mean pore diameter of about $24 \mathrm{~nm}$, prepared using a sol-gel method and low-cost silica precursors, was immersed in the YAG:Tb sol. The impregnation process was followed by a drying step and an annealing process at $1100{ }^{\circ} \mathrm{C}$ for $4 \mathrm{~h}$. XRD analysis and HRTEM images have obviously shown the formation of highly crystalline YAG:Tb nanocrystals with the YAG cubic structure and a crystallite average diameter of about $23 \mathrm{~nm}$. The results also showed a uniform dispersion of YAG:Tb nanocrystals in the MSM matrix, leading to a strong and homogeneous green emission from the composite. The PL properties of the MSM-YAG:Tb composite have been discussed and compared to those of neat YAG:Tb phosphor. It was found that excitation spectra are shifted to a lower wavelength and the PL decay time is significantly shorter in the case of the MSM-YAG:Tb composite compared to the neat YAG:Tb phosphor. Moreover, PL emission spectra of the MSM-YAG:Tb nanocomposite showed broader emission bands when compared to those of the YAG:Tb powder. These results are in line with the formation of a nano-sized YAG:Tb phosphor within the MSM host matrix. The method we developed in the present work can obviously be applied, as a versatile procedure, to a large variety of materials (oxides, fluorides, etc.) synthesized by the sol-gel route. A large size $(30 \times 100 \mathrm{~mm})$ sculpted MSMYAG:Tb composite was successfully designed and has the potential to be applied to decorative lighting.

\section{Acknowledgements}

The authors thank Elisabeth Hans (Jewellery, creation and design realization) for the sculpture realized on the MSM-YAG:Tb piece (www.ehans-joailler.com). Research on sol-gel silica monoliths was partly supported by the "Fonds Européen de Développement Economique Régional" and the Labex CEMPI and Equipex FLUX 
through the "Programme Investissements d'Avenir". The TEM facility in Lille (France) is supported by the Conseil Régional du Nord-Pas de Calais and the European Regional Development Fund (ERDF).

\section{Notes and references}

1 S. Shionoya, W. M. Yen and H. Yamamoto, Phosphor Handbook, CRC Press, 2006.

2 C. C. Lin and R.-S. Liu, J. Phys. Chem. Lett., 2011, 2, 1268-1277.

3 S. Ye, F. Xiao, Y. X. Pan, Y. Y. Ma and Q. Y. Zhang, Mater. Sci. Eng., R, 2010, 71, 1-34.

4 A. Aboulaich, M. Michalska, R. Schneider, A. Potdevin, J. Deschamps, R. Deloncle, G. Chadeyron and R. Mahiou, ACS Appl. Mater. Interfaces, 2014, 6, 252-258.

5 Y.-C. Lin, J. P. You, N. T. Tran, Y. He and F. G. Shi, J. Electron. Packag., 2011, 133, 011009.

6 N. Bardsley, S. Bland, D. Chwastyk, C. D. Monasterio, L. Pattison, M. Pattison, F. Welsh and M. Yamada, Solidstate Lighting Research and Development, Manufacturing Roadmap, US Department of Energy, 2013.

7 S. C. Huang, J. K. Wu, W.-J. Hsu, H. H. Chang, H. Y. Hung, C. L. Lin, H.-Y. Su, N. Bagkar, W.-C. Ke, H. T. Kuo and R.-S. Liu, Int. J. Appl. Ceram. Technol., 2009, 6, 465-469.

8 L. Chen, C.-I. Chu and R.-S. Liu, Microelectron. Reliab., 2012, 52, 900-904.

9 A. Aboulaich, J. Deschamps, R. Deloncle, A. Potdevin, B. Devouard, G. Chadeyron and R. Mahiou, New J. Chem., 2012, 36, 2493-2500.

10 D. Jia, Chem. Eng. Commun., 2007, 194, 1666-1687.

11 D. Jia, Electrochem. Solid-State Lett., 2006, 9, H93-H95.

12 D. Jia, Y. Wang, X. Guo, K. Li, Y. K. Zou and W. Jia, J. Electrochem. Soc., 2007, 154, J1-J4.

13 L. T. Su, A. I. Y. Tok, Y. Zhao, N. Ng and F. Y. C. Boey, J. Phys. Chem. B, 2009, 113, 5974-5979.

14 N. Pradal, G. Chadeyron, A. Potdevin, J. Deschamps and R. Mahiou, J. Eur. Ceram. Soc., 2013, 33, 1935-1945.

15 S. Das, A. Amarnath Reddy, S. Ahmad, R. Nagarajan and G. Vijaya Prakash, Chem. Phys. Lett., 2011, 508, 117-120.

16 A. Bao, H. Yang and C. Tao, Curr. Appl. Phys., 2009, 9, 1252-1256.

17 S. S. Pitale, V. Kumar, I. Nagpure, O. M. Ntwaeaborwa and H. C. Swart, Curr. Appl. Phys., 2011, 11, 341-345.

18 J. Liao, H. You, B. Qiu, H.-R. Wen, R. Hong, W. You and Z. Xie, Curr. Appl. Phys., 2011, 11, 503-507.

19 F. Hoffmann, M. Cornelius, J. Morell and M. Fröba, Angew. Chem., Int. Ed., 2006, 45, 3216-3251.

20 S. J. Lee, S. H. Park Ss Fau - Lee, S.-H. Lee Sh Fau - Hong, C.-S. Hong Sh Fau - Ha and C. S. Ha, J. Nanosci. Nanotechnol., 2013, 13, 7459-7466.

21 A. Feinle, F. Lavoie-Cardinal, J. Akbarzadeh, H. Peterlik, M. Adlung, C. Wickleder and N. Hüsing, Chem. Mater., 2012, 24, 3674-3683.

22 N. Brun, B. Julián-López, P. Hesemann, G. Laurent, H. Deleuze, C. Sanchez, M.-F. Achard and R. Backov, Chem. Mater., 2008, 20, 7117-7129.
23 Y. Du, Y. Fu, X. Guo, H. Li, C. Lü and Z. Su, Microporous Mesoporous Mater., 2010, 130, 122-129.

24 Q. Meng, L. Fu, J. Lin, H. Zhang, S. Wang, Y. Zhou, M. Yu and F. Liu, J. Phys. Chem. Solids, 2003, 64, 63-67.

25 C. Stan, N. Marcotte, M. Secula and M. Popa, J. Sol-Gel Sci. Technol., 2014, 69, 207-213.

26 X. Gao and S. Nie, J. Phys. Chem. B, 2003, 107, 11575-11578.

27 J. Yang, Y. Deng, Q. Wu, J. Zhou, H. Bao, Q. Li, F. Zhang, F. Li, B. Tu and D. Zhao, Langmuir, 2010, 26, 8850-8856.

28 L. Sorensen, G. F. Strouse and A. E. Stiegman, Adv. Mater., 2006, 18, 1965-1967.

29 Y. Chen, Q. Chen, L. Song, H.-P. Li and F.-Z. Hou, Microporous Mesoporous Mater., 2009, 122, 7-12.

30 Y. J. Gu and B. Yan, J. Colloid Interface Sci., 2013, 393, 36-43.

31 Y. Chen, Q. Chen, L. Song, H.-p. Li and F.-z. Hou, J. Alloys Compd., 2010, 490, 264-269.

32 H. Xu, L. Cheng, C. Wang, X. Ma, Y. Li and Z. Liu, Biomaterials, 2011, 32, 9364-9373.

33 Y.-F. Han, F. Chen, Z. Zhong, K. Ramesh, L. Chen and E. Widjaja, J. Phys. Chem. B, 2006, 110, 24450-24456.

34 T. Takayuki, H. Manabu, D. Hiroyuki, M. Masaki, K. Atsushi and K. Shigemi, Jpn. J. Appl. Phys., 2010, 49, 06GH04.

35 G. D. Mihai, V. Meynen, M. Mertens, N. Bilba, P. Cool and E. F. Vansant, J. Mater. Sci., 2010, 45, 5786-5794.

36 Z. Li, H. Zhang and H. Fu, J. Lumin., 2013, 135, 79-83.

37 A. Potdevin, G. Chadeyron, D. Boyer and R. Mahiou, J. Mater. Sci., 2006, 41, 2201-2209.

38 H. El Hamzaoui, L. Courthéoux, V. N. Nguyen, E. Berrier, A. Favre, L. Bigot, M. Bouazaoui and B. Capoen, Mater. Chem. Phys., 2010, 121, 83-88.

39 A. Potdevin, G. Chadeyron and R. Mahiou, Chem. Phys. Lett., 2010, 490, 50-53.

40 S. Brunauer, P. H. Emmett and E. Teller, J. Am. Chem. Soc., 1938, 60, 309-319.

41 E. P. Barrett, L. G. Joyner and P. P. Halenda, J. Am. Chem. Soc., 1951, 73, 373-380.

42 B. D. Cullity and S. R. Stock, Elements of X-Ray Diffraction, Prentice Hall, 3rd edn, 2001.

43 A. Nakatsuka, A. Yoshiasa and T. Yamanaka, Acta Crystallogr., Sect. B: Struct. Sci., 1999, 55, 266-272.

44 J. A. Koningstein, Chem. Phys. Lett., 1969, 3, 303-304.

45 O. Yamaguchi, K. Takeoka, K. Hirota, H. Takano and A. Hayashida, J. Mater. Sci., 1992, 27, 1261-1264.

46 P. Padmaja, G. M. Anilkumar, P. Mukundan, G. Aruldhas and K. G. K. Warrier, Int. J. Inorg. Chem., 2001, 3, 693-698.

47 P. Kansal, R. M. Laine and F. Babonneau, J. Am. Ceram. Soc., 1997, 80, 2597-2606.

48 A. Potdevin, G. Chadeyron, V. Briois, F. Leroux and R. Mahiou, Dalton Trans., 2010, 39, 8718-8724.

49 N. Bodenschatz, R. Wannemacher, J. Heber and D. Mateika, J. Lumin., 1990, 47, 159-167.

50 H.-h. Kwak, S.-J. Kim, H.-H. Yoon, S.-J. Park and H.-w. Choi, J. Electroceram., 2009, 23, 397-401.

51 J. Zhou, F. Zhao, X. Wang, Z. Li, Y. Zhang and L. Yang, J. Lumin., 2006, 119-120, 237-241. 
52 M. Kubus, H. J. r. Meyer, L. Kienle and A. M. Klonkowski, J. Non-Cryst. Solids, 2009, 355, 1333-1337.

53 A. Potdevin, G. Chadeyron, D. Boyer and R. Mahiou, J. Appl. Phys., 2007, 102, 073536.

54 J. Y. Choe, D. Ravichandran, S. M. Blomquist, K. W. Kirchner, E. W. Forsythe and D. C. Morton, J. Lumin., 2001, 93, 119-128.

55 Z. Zhang, O. M. ten Kate, A. Delsing, E. van der Kolk, P. H. L. Notten, P. Dorenbos, J. Zhao and H. T. Hintzen, J. Mater. Chem., 2012, 22, 9813-9820.

56 Y. Hakuta, T. Haganuma, K. Sue, T. Adschiri and K. Arai, Mater. Res. Bull., 2003, 38, 1257-1265.
57 S. Zhou, Z. Fu, J. Zhang and S. Zhang, J. Lumin., 2006, 118, 179-185.

58 P. Packiyaraj and P. Thangadurai, J. Lumin., 2014, 145, 997-1003.

59 H. Peng, H. Song, B. Chen, J. Wang, S. Lu, X. Kong and J. Zhang, J. Phys. Chem., 2003, 118, 3277-3282.

60 R. Hansel, S. Allison and G. Walker, MRS Online Proc. Libr., 2008, 1076, K06.

61 R. S. Meltzer, S. P. Feofilov, B. Tissue and H. B. Yuan, Phys. Rev. B: Condens. Matter Mater. Phys., 1999, 60, R14012-R14015. 